\title{
Impact of Magnetic Field Configuration on Heat Transport in Stellarators and Heliotrons
}

\author{
Felix Warmer $\odot,{ }^{1, *}$ K. Tanaka, ${ }^{2,3}$ P. Xanthopoulos, ${ }^{1}$ M. Nunami, ${ }^{2,45}$ M. Nakata, ${ }^{2,4}$ C. D. Beidler, ${ }^{1}$ S. A. Bozhenkov, ${ }^{1}$ \\ M. N. A. Beurskens, ${ }^{1}$ K. J. Brunner, ${ }^{1}$ O. P. Ford, ${ }^{1}$ G. Fuchert, ${ }^{1}$ H. Funaba, ${ }^{2}$ J. Geiger, ${ }^{1}$ D. Gradic, ${ }^{1}$ K. Ida ${ }^{2,4}$ H. Igami, ${ }^{2}$ \\ S. Kubo, ${ }^{2,5}$ A. Langenberg, ${ }^{1}$ H. P. Laqua, ${ }^{1}$ S. Lazerson, ${ }^{1}$ T. Morisaki, ${ }^{2,4}$ M. Osakabe ${ }^{2,4}$ N. Pablant, ${ }^{6}$ E. Pasch, ${ }^{1}$ B. Peterson, ${ }^{2}$ \\ S. Satake, ${ }^{2,4}$ R. Seki, ${ }^{2,4}$ T. Shimozuma, ${ }^{2}$ H. M. Smith, ${ }^{1}$ T. Stange, ${ }^{1}$ A. v. Stechow, ${ }^{1}$ H. Sugama, ${ }^{2,4}$ Y. Suzuki, ${ }^{2,4}$ \\ H. Takahashi, ${ }^{2,4}$ T. Tokuzawa, ${ }^{2,4}$ T. Tsujimura, ${ }^{2,4}$ Y. Turkin, ${ }^{1}$ R. C. Wolf, ${ }^{1}$ I. Yamada, ${ }^{2}$ R. Yanai, ${ }^{2}$ R. Yasuhara, ${ }^{2,4}$ \\ M. Yokoyama, ${ }^{2,4}$ Y. Yoshimura, ${ }^{2}$ M. Yoshinuma, ${ }^{2,4}$ D. Zhang, ${ }^{1}$ W7-X Team, ${ }^{1}$ and LHD Experimental Group ${ }^{2}$ \\ ${ }^{1}$ Max-Planck-Institut für Plasmaphysik, Wendelsteinstrasse 1, 17491 Greifswald, Germany \\ ${ }^{2}$ National Institute for Fusion Science, National Institutes on Natural Sciences, Toki, 509-5292, Japan \\ ${ }^{3}$ Kyushu University, Interdisciplinary Graduate School of Engineering Sciences, \\ Plasma and Quantum Science and Engineering, Kasuga, Fukuoka 816-8580, Japan \\ ${ }^{4}$ SOKENDAI (The Graduate University for Advanced Studies), Toki, Gifu 509-5292, Japan \\ ${ }^{5}$ Nagoya University, Graduate School of Science, Nagoya 464-8603, Japan \\ ${ }^{6}$ Princeton Plasma Physics Laboratory, Princeton, New Jersey 08543, USA
}

(Received 31 March 2021; revised 30 July 2021; accepted 20 September 2021; published 24 November 2021)

\begin{abstract}
We assess the magnetic field configuration in modern fusion devices by comparing experiments with the same heating power, between a stellarator and a heliotron. The key role of turbulence is evident in the optimized stellarator, while neoclassical processes largely determine the transport in the heliotron device. Gyrokinetic simulations elucidate the underlying mechanisms promoting stronger ion scale turbulence in the stellarator. Similar plasma performances in these experiments suggests that neoclassical and turbulent transport should both be optimized in next step reactor designs.
\end{abstract}

DOI: 10.1103/PhysRevLett.127.225001

Introduction.-Stellarators and heliotrons are toroidal magnetic confinement configurations that generate their magnetic fields by relying on external coils. This feature allows for the existence of flux surfaces in vacuum and offers steady state operation capability. Furthermore, these fusion devices are far less susceptible than axisymmetric tokamaks to instabilities associated with large plasma currents, which could jeopardize the structural integrity of the torus. Early designs suffered from lack of confinement for particles deeply trapped inside the local minima caused by the nonaxisymmetric corrugation of the magnetic field. Such radially diffusing particles are responsible for the so-called "neoclassical transport," which is exacerbated by high temperatures. A breakthrough in stellarator research has been the reduction of neoclassical transport via shaping of the magnetic geometry [1-4]. The quantity that characterizes the degree of neoclassical optimization is the effective helical ripple, $\epsilon_{\text {eff }}$ [5], representing the helical ripple amplitude that is required to obtain the same level of

Published by the American Physical Society under the terms of the Creative Commons Attribution 4.0 International license. Further distribution of this work must maintain attribution to the author(s) and the published article's title, journal citation, and DOI. Open access publication funded by the Max Planck Society. neoclassical transport in a simple model stellarator. The reduction of $\epsilon_{\text {eff }}$ has been, in fact, the cornerstone of the Wendelstein 7-X (W7-X) stellarator design [6]. Another contender for fusion energy is the large helical device (LHD) [7], originally designed to demonstrate the functionality of superconducting helical coils for long pulse operation, and the negative ion neutral beam heating, in order to achieve high plasma pressures. Although its magnetic field has not been explicitly optimized, the "inward shifted" configuration of LHD, with magnetic axis position $R_{\mathrm{ax}}=3.6 \mathrm{~m}$, has a reduced neoclassical transport relative to the configuration with $R_{\mathrm{ax}}>3.6 \mathrm{~m}$.

The plasma shape and principal geometric properties for the two devices are illustrated in Fig. 1. In addition, as inferred from Fig. 2 (left), the W7-X magnetic field is characterized by low magnetic shear $(d l / d \rho)$. This helps to avoid low order rationals in the rotational transform, $l / 2 \pi$, across the plasma center, that would produce undesirable magnetic islands. Simultaneously, a magnetic resonance at the edge plasma region is formed, which enables island divertor operation. The LHD heliotron, in contrast, features a strong magnetic shear, which inhibits the formation of magnetic islands and stabilizes interchange type modes (we note that, for both devices, the magnetic shear is directed opposite to that in tokamaks). In Fig. 2 (right), we also show that $\epsilon_{\text {eff }}$ for the standard W7-X configuration is around $1 \%$ across the entire plasma radius. To compare, 


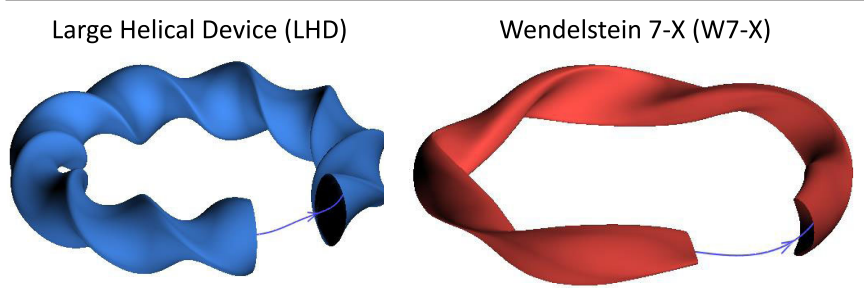

FIG. 1. (Left) The large helical device is a heliotron, featuring an elliptic plasma cross section that rotates around the torus for a total of 10 field periods, with major radius $R=3.6 \mathrm{~m}$ and aspect ratio $A=5.5$. (Right) The Wendelstein 7-X (W7-X) stellarator has a helical plasma axis with a varying cross section in the toroidal direction for a total of 5 field periods, with major radius $R=5.5 \mathrm{~m}$ and aspect ratio $A=10$. The plasma volume in both configurations measures approximately 30 cubic meters.

for the inward shifted LHD configuration, $\epsilon_{\text {eff }}$ is strongly increasing from the plasma core, to reach almost $6 \%$ towards the plasma edge.

It follows that in LHD, the neoclassical transport can account for a large portion of the total transport, depending on the radial electric field strength, the magnetic configuration [8] and density regime $[9,10]$. Regarding turbulent transport, theoretical studies show that LHD configurations with small $\epsilon_{\text {eff }}$, like the inward shifted configuration, have the advantage of relatively low turbulent losses due to larger zonal flow generation $[11,12]$. On the other hand, in W7-X, turbulent processes can under certain heating conditions [13] dominate the heat transport, thus limiting the plasma performance despite the field optimization. In this context, the question arises naturally, whether controlling either the neoclassical or the turbulent transport would be an effective strategy to enhance the plasma confinement. We tackle this question using power balance analysis combined with numerical simulations from tailored experiments in LHD and W7-X, as described below.

Experimental setup and plasma profiles.-The discharges for the LHD experiment No. 152264 and the W7-X program No. 20180821.017 are designed by employing 2 megawatts of electron cyclotron resonance (ECR) power [14-16] introduced into each device, with the ECR absorption located slightly of axis in LHD. As a result
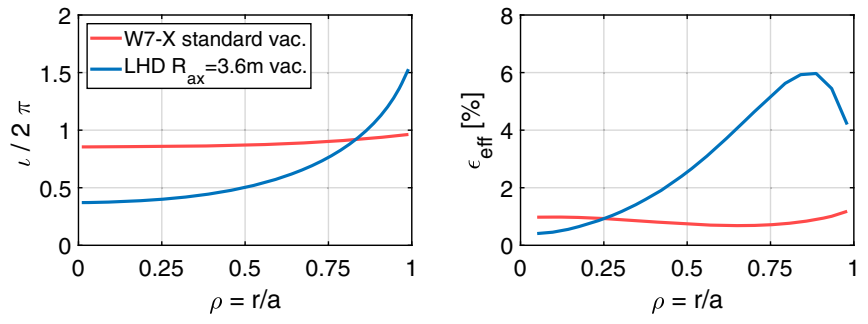

FIG. 2. Rotational transform $\imath / 2 \pi$ (left) and effective helical ripple $\epsilon_{\text {eff }}$ (right) along the plasma radius, for the $\mathrm{W} 7-\mathrm{X}$ stellarator in its standard vacuum configuration and the LHD heliotron in its inward shifted vacuum configuration. of this heating scheme, a moderate line averaged density $\left\langle n_{e}\right\rangle \sim 1.5 \times 10^{19} \mathrm{~m}^{-3}$ is achieved. The two discharges feature similar plasma performances, with the confinement time for the LHD experiment $(160 \mathrm{msec})$ being somewhat larger than that for the W7-X program $(120 \mathrm{msec})$. Interestingly, despite these similarities, the measured profiles are strikingly different, as evidenced by Fig. 3. Indeed, the electron density profile $n_{e}$ is almost flat for W7-X [17] in the plasma core, whereas for LHD it exhibits a hollow shape $[18,19]$. Furthermore, the ion temperature $T_{i}$ reaches comparable values in the plasma center for the two devices, although LHD tends to sustain higher ion temperatures in the plasma periphery. The electron temperature $T_{e}$ is peaked in the W7-X core due to the strongly localized ECR heating, and it falls off rapidly towards the plasma edge. In $\mathrm{LHD}, T_{e}$ is less peaked, and retains relatively large values towards the plasma edge.

Power balance and simulations.-The disparity between the plasma profiles in the two experiments is an indicator of different dominant transport processes. We, therefore, conduct a power balance analysis for the characterization of the transport, focusing on the ions as the fusion relevant particle species. Our goal is to extract the neoclassical and turbulent contributions from the total ion heat transport. Using the measured density and temperature profiles, the neoclassical ion energy flux $Q_{i}^{\mathrm{NC}}$ is
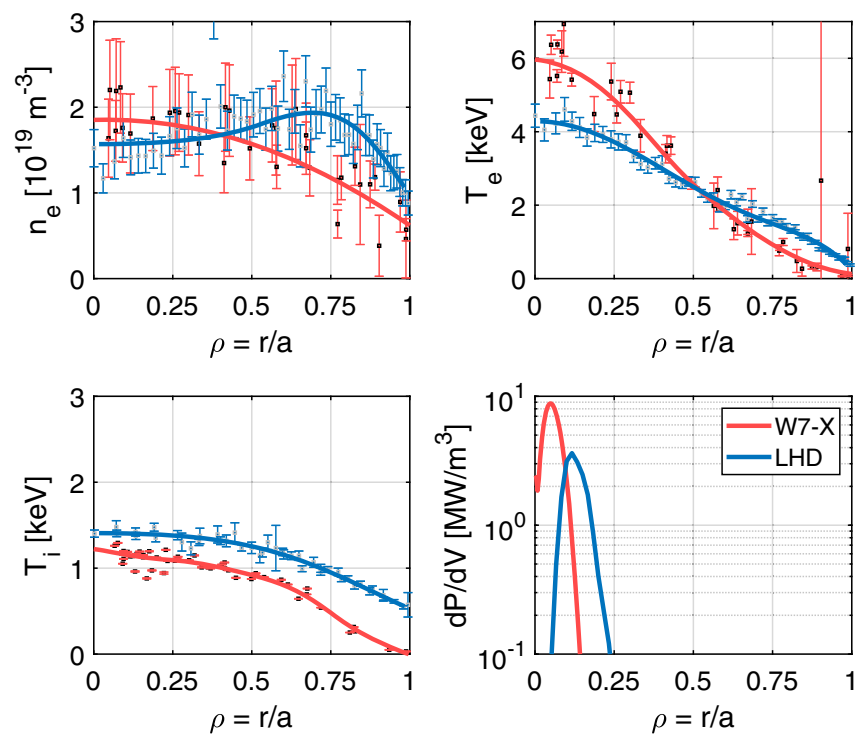

FIG. 3. Profiles of electron density $n_{e}$, electron temperature $T_{e}$, ion temperature $T_{i}$, and absorbed ECR heating power along the plasma radius for the LHD experiment No. 152264 at $t=4.47 \mathrm{sec}$ and the W7-X program No. 20180821.017 at $t=4.30 \mathrm{sec} . T_{e}$ and $n_{e}$ were measured with Thomson scattering diagnostics [20,21]. In LHD, $n_{e}$ was also measured using a multichannel far infrared interferometer system [22]. $T_{i}$ and the radial electric field $E_{r}$ in LHD were measured with active charge exchange recombination spectroscopy (CXRS), using short $20 \mathrm{~ms}$ blips of perpendicular neutral beam injection [23]. In W7-X, $T_{i}$ was also measured with a CXRS system [24]. 

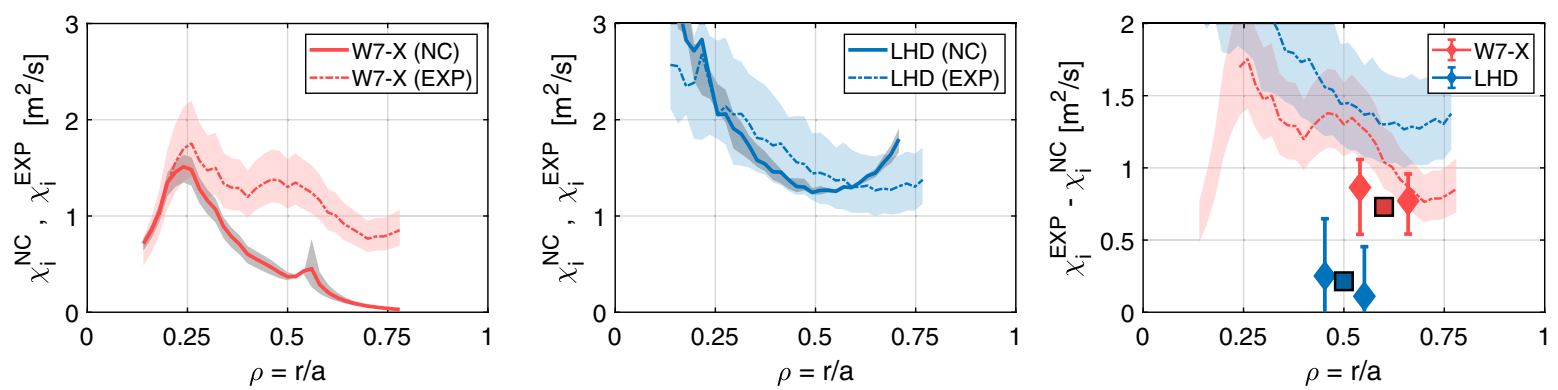

FIG. 4. Experimental (dashed dotted lines) and neoclassical (solid lines) ion heat conductivities for W7-X (left) and LHD (middle), and anomalous heat conductivities (diamonds, right), as extracted from the power balance analysis. The squares (right) correspond to the simulated values of the heat transport. The experimental heat conductivities (dashed dotted lines) are repeated in the right panel for comparison. The shaded areas reflect uncertainties stemming from the error bars of the plasma profiles.

calculated using the code DKES [25,26], which solves the monoenergetic linearized drift kinetic equation as a function of a set of dimensionless quantities (flux surface, collisionality and $\mathbf{E} \times \mathbf{B}$ drift velocity). The transport coefficients are subsequently obtained by an energy convolution over the Maxwellian distribution. The remaining energy losses, referred to as "anomalous transport," are thought to be caused by plasma turbulence. The results of the power balance analysis are summarized in Fig. 4. In accordance with the magnetic field optimization, the neoclassical ion diffusivity $\chi_{i}^{\mathrm{NC}}$ is much lower for the W7-X stellarator compared to that for the LHD heliotron. A remarkable feature, however, is that the anomalous ion diffusivity $\chi_{i}^{\mathrm{ANO}}$, defined as $\chi_{i}^{\mathrm{EXP}}-\chi_{i}^{\mathrm{NC}}$, is significantly larger for the optimized W7-X stellarator.

In order to evaluate the relative strength of the anomalous transport in the two devices, we first identify the relevant type of small scale turbulence that can be excited in such plasmas [27]. To this end, we employ two numerical solvers of the nonlinear set of gyrokinetic equations [28]: The GENE code [29], for the simulations in the W7-X stellarator, and the GKV code [30-32], for the simulations in the LHD heliotron. The gyrokinetic solvers are operated in the "flux tube" geometry [33], namely, we construct a slender computational box along a single magnetic field line on a flux surface. For the W7-X simulations, we select the surface $\rho=r / a=0.6$ and the field line $\alpha=0$ ( $\alpha=q \theta-\zeta$, where $\theta$ is the poloidal angle of the torus, $\zeta$ is the toroidal angle and $q$ is the safety factor). For the LHD simulations, we select the surface $\rho=0.5$ and the field line $\alpha=\pi / 10$. [We note in passing that the slightly different radial location is dictated by the disparity of the profiles in the two configurations; we expect stronger turbulence at $\rho=0.5$ for LHD compared to $\rho=0.6$.] In each configuration, the flux tube crosses the region of most unfavorable curvature on the surface within one poloidal turn. According to the measured profiles, the normalized ion temperature and density gradients, and the electron to ion temperature ratio for W7-X read $a / L_{T_{i}}=-a / T_{i} d T_{i} / d r=2.0, a / L_{n}=1.07$ and
$T_{e} / T_{i}=2.28$, whereas for LHD they read $a / L_{T_{i}}=0.65$, $a / L_{n}=-0.63$ and $T_{e} / T_{i}=2$. The linear gyrokinetic calculations reveal that the most prominent instability for both experiments, at the radii of interest, is ion temperature gradient (ITG) driven [10,34]. The associated growth rates, shown in Fig. 5 (inset), suggest that the ITG instability is much stronger in the W7-X stellarator. Using these parameters, we also performed nonlinear gyrokinetic simulations, in order to estimate the ITG turbulence in the two configurations, treating both ions and electrons as gyrokinetic species. Simplifications in the simulations settings are thought to have a minor impact. For instance, both plasmas have very low beta values $(0.23 \%$ in $\mathrm{W} 7-\mathrm{X}$ and $0.28 \%$ in LHD) and are found in the low collisionality regime for

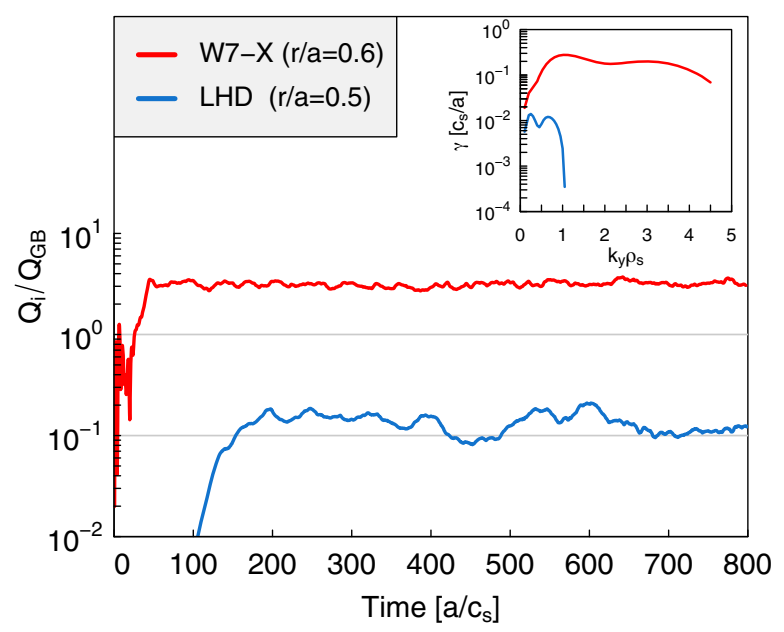

FIG. 5. Ion heat fluxes, normalized to gyroBohm units $Q_{G B}=\rho_{s}^{2} c_{s} P_{i} / a^{2}$, inferred from turbulence simulations for W7-X and LHD, according to the measured profiles $\left(P_{i}\right.$ is the ion pressure, $a$ is the minor radius, $\rho_{s}=c_{s} / \Omega_{i}$ is the ion gyroradius, $c_{s}=\sqrt{T_{i} / m_{i}}$ is the ion sound speed, $\Omega_{i}=$ $q B / m_{i} c$ is the ion gyrofrequency, $B$ is the magnetic field, $q$ is the ion charge and $m_{i}$ is the ion mass). The inset figure shows the growth rates of the ITG instability as a function of the binormal wave number normalized to the ion gyroradius. 
the entire plasma volume. These conditions justify to a large degree the treatment of the plasma as electrostatic and collisionless for our simulations. In addition, at radius $\rho=0.6$, the neoclassical radial electric field in $\mathrm{W} 7-\mathrm{X}$ is close to zero due to the field changing its sign. For LHD, the poloidal Mach number is larger, but nevertheless stays below $M_{p}=0.15$. Numerical simulations for the inward shifted configuration, considering twice as high $M_{p}$, have demonstrated that the effect of the radial electric field on ITG turbulence is minimal [35]. The outcome of the gyrokinetic nonlinear simulations, shown in Fig. 5, provides the ion heat fluxes, normalized to gyroBohm units. We find that the average ion heat flux for W7-X is over an order of magnitude larger than that for LHD. Converting to ion heat diffusivities, the simulated average values are $\chi_{\mathrm{i}, \text { sim }}^{\mathrm{W} \text { 7X }}=0.732\left(\mathrm{~m}^{2} / \mathrm{sec}\right)$ and $\chi_{\mathrm{i}, \mathrm{sim}}^{\mathrm{LHD}}=0.214\left(\mathrm{~m}^{2} / \mathrm{sec}\right)$. A comparison against the experimental values of the ion heat diffusivity is shown in Fig. 4 (right panel).

Although we have identified the weak instability as the main reason for the lower heat transport in the LHD heliotron, in the following, we also examine effects that might play a secondary, but still significant, role. Specifically, we investigate the role of the zonal flows [36] for the turbulence saturation. It is already known that ITG turbulence is significantly affected by the zonal flows in the W7-X [37] stellarator as well as the LHD heliotron [38-40]. The strength of the zonal flows produced in a magnetic configuration is dependent on the turbulence drive. In general, the stronger the drive becomes, the more zonal flow content is necessary to achieve saturation. Therefore, in order to better assess the relative contribution of the zonal flows to the turbulent transport in each device, we prefer to follow a generic approach, using a more simplified set of parameters than that dictated by the profiles, namely we apply for both devices $a / L_{T_{i}}=3$, also setting $a / L_{n}=0$ and $T_{e} / T_{i}=1$. These parameters remain, however, realistic, and can be reproduced by most of the discharges of similar type, especially towards the plasma periphery. The electrons in these simulations are considered adiabatic. From the nonlinear simulations, shown in Fig. 6, we deduce that the shearing effect imposed on the turbulence eddies is more efficient in the heliotron device, by employing a specific figure of merit. This is defined as the ratio $\gamma_{\max } / \Omega_{E}$, where the growth rate $\gamma_{\max }$ is maximized over the poloidal wave number, and $\Omega_{E}=\hat{B} V_{E}^{\prime}$ is the shearing rate, defined through the radial derivative of the $\mathbf{E} \times \mathbf{B}$ velocity $V_{E}$ of the zonal flow $(\hat{B}$ is the normalized magnetic field modulus). We extract from the simulations that the ratio is significantly smaller for LHD (0.018) compared to W7-X (0.230), suggesting that the zonal flows should be more important for the saturation of ITG turbulence in LHD compared to W7-X. A plausible explanation for the stronger impact of the zonal flows in LHD can be based on the localization of the density fluctuations along the flux tube. Since the turbulence in LHD appears less localized

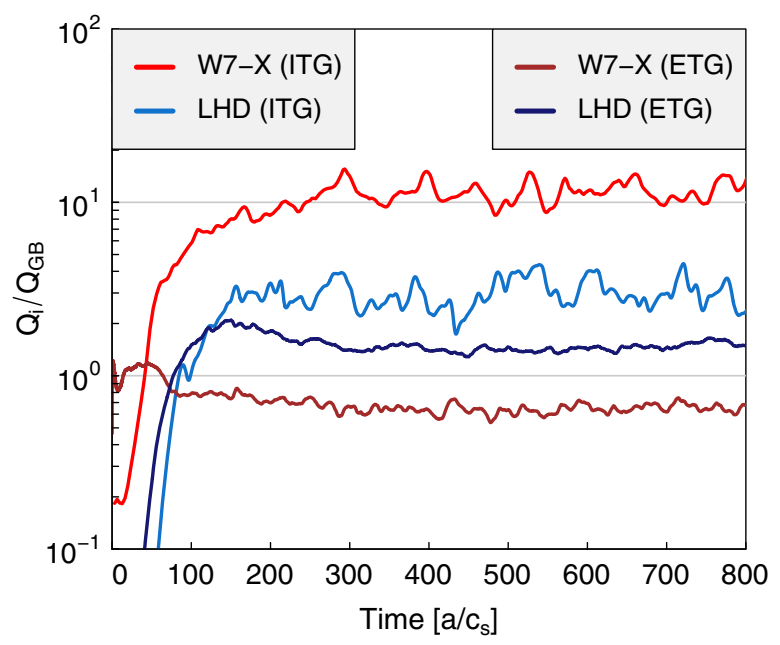

FIG. 6. Ion heat fluxes, normalized to gyroBohm units (for definitions, see Fig. 5), inferred from turbulence simulations for W7-X and LHD. For the ITG simulations, we have used the same normalized ion temperature gradient $a / L_{T_{i}}=3$ and adiabatic electrons, whereas for the ETG simulations, we have used the same electron temperature gradient $a / L_{T_{e}}=3$ and adiabatic ions. For all simulations, $a / L_{n}=0$ and $T_{e} / T_{i}=1$ is assumed.

compared to that in W7-X, this implies that the space filling factor [37], which directly enters the nonlinear zonal flow drive, should be larger in LHD.

Furthermore, we have performed gyrokinetic simulations for the electron temperature gradient (ETG) driven turbulence, applying gradients similar to the ITG simulations, i.e., $a / L_{T_{e}}=3, a / L_{n}=0$ and $T_{e} / T_{i}=1$. The ETG simulations are conducted assuming adiabatic ions. Since ETG turbulence hardly responds to the zonal flows [41], the turbulence level may relate only to the geometric properties of the configuration, such as the local magnetic curvature. In Fig. 6, we show that the normalized ETG heat flux for the W7-X stellarator is lower than that for the LHD heliotron. From this observation, we expect that, in the absence of zonal flows, the ITG simulation would predict also a lower ion heat flux for W7-X. The fact, however, that the ion heat flux for the W7-X is instead larger, emphasizes the key role of zonal flows for the ITG turbulence saturation, particularly for LHD.

Conclusions.-Conducting low-density, low-power ECR heated experiments on two large fusion facilities, namely, the optimized stellarator Wendelstein 7-X (W7-X) and the large helical device (LHD), combined with gyrokinetic simulations, we conclude that quite distinct transport processes may lead to similar plasma performances. In the LHD heliotron, the neoclassical transport appears to dominate over the entire plasma radius, while the turbulent transport is relatively benign. On the other hand, in the optimized W7-X stellarator, the neoclassical contribution to the transport is much lower than that for the LHD heliotron, and turbulence emerges as an important factor for the anomalous ion heat losses. In addition to the larger growth 
rate for the ITG mode, we have identified the zonal flows as a plausible reason for the stronger ITG turbulence in the W7-X stellarator compared to the LHD heliotron. The figure of merit that represents the relative strength of zonal flows, expressed by the ratio $\gamma_{\max } / \Omega_{E}$, is found to be much smaller in the heliotron device, hinting at a more effective shearing of the turbulence eddies.

Our work suggests that a next step reactor sized stellarator design should address both neoclassical and turbulent transport, through shaping of the magnetic field geometry. Preliminary attempts have demonstrated the feasibility of this approach, applying a global search optimization scheme in the W7-X configuration space [42-44]. Meanwhile, current experiments should continue to explore favorable plasma scenarios. For instance, injections of frozen hydrogen pellets in W7-X [45] seem to suppress ITG turbulence thanks to the steep density gradient, which is typically induced by the fuel [46]. As a result, enhanced performances have been achieved, exceeding the predictions from the empirical ISS04 scaling [47].

The support from the technical and engineering staff of the LHD and W7-X experimental groups is greatly appreciated. The simulations were performed on JFRS-1 at the International Fusion Energy Research Centre and the Plasma Simulator at NIFS (Japan), the Rechenzentrum Garching supercomputer (Germany), and the Marconi supercomputer (Italy). This work is supported by NIFS Grants NIFS18ULHH013, NIFS18KLER045, NIFS18KLPH032, NIFS18KUHL083, NIFS20KNST161 and JSPS Grant 16H04620, 20K03907, 17K14899, as well as by PLADyS, JSPS Core-to-Core Program, A. Advanced Research Networks. This work has been carried out within the framework of the EUROfusion Consortium and has received funding from the Euratom research and training programme 2014-2018 and 2019-2020 under Grant Agreement No. 633053. The views and opinions expressed herein do not necessarily reflect those of the European Commission.

*Corresponding author.

Felix.Warmer@ipp.mpg.de

[1] J. Nührenberg and R. Zille, Quasi-helically symmetric toroidal stellarators, Phys. Lett. A 129, 113 (1988).

[2] A. A. Subbotin, M. I. Mikhailov, V. D. Shafranov, M. Y. Isaev, C. Nührenberg, J. Nührenberg, R. Zille, V. V. Nemov, S. V. Kasilov, V. N. Kalyuzhnyj, and W. A. Cooper, Integrated physics optimization of a quasi-isodynamic stellarator with poloidally closed contours of the magnetic field strength, Nucl. Fusion 46, 921 (2006).

[3] C. D. Beidler, K. Allmaier, M. Y. Isaev, S. V. Kasilov, W. Kernbichler, G. O. Leitold, H. Maaßberg, D. R. Mikkelsen, S. Murakami, M. Schmidt, D. A. Spong, V. Tribaldos, and A. Wakasa, Benchmarking of the mono-energetic transport coefficients-results from the International Collaboration on Neoclassical Transport in Stellarators (ICNTS), Nucl. Fusion 51, 076001 (2011).

[4] H. E. Mynick, Transport optimization in stellarators, Phys. Plasmas 13, 058102 (2006).

[5] C. Beidler and H. Maaßberg, An improved formulation of the ripple-averaged kinetic theory of neoclassical transport in stellarators, 43, 1131 (2001).

[6] T. Klinger et al., Overview of first Wendelstein 7-X highperformance operation, Nucl. Fusion 59, 112004 (2019).

[7] Y. Takeiri et al., Extension of the operational regime of the LHD towards a deuterium experiment, Nucl. Fusion 57, 102023 (2017).

[8] K. Ida et al., Reduction of Ion Thermal Diffusivity Associated with the Transition of the Radial Electric Field in Neutral-Beam-Heated Plasmas in the Large Helical Device, Phys. Rev. Lett. 86, 5297 (2001).

[9] F. Warmer et al., Energy confinement of hydrogen and deuterium electron-root plasmas in the Large Helical Device, Nucl. Fusion 58, 106025 (2018).

[10] K. Tanaka et al., Extended investigations of isotope effects on ECRH plasma in LHD, 62, 024006 (2020).

[11] H. Sugama and T.-H. Watanabe, Dynamics of Zonal Flows in Helical Systems, Phys. Rev. Lett. 94, 115001 (2005).

[12] T.-H. Watanabe, H. Sugama, and S. Ferrando-Margalet, Reduction of Turbulent Transport with Zonal Flows Enhanced in Helical Systems, Phys. Rev. Lett. 100, 195002 (2008).

[13] M. N. A. Beurskens et al., Ion temperature clamping in Wendelstein 7-X Electron Cyclotron Heated plasmas, Nucl. Fusion 61, 116072 (2021).

[14] H. Igami, S. Kubo, T. Shimozuma, Y. Yoshimura, H. Takahashi, S. Kamio, S. Kobayashi, S. Ito, Y. Mizuno, K. Okada, R. Makino, S. Ogasawara, K. Kobayashi, M. Osakabe, K. Nagasaki, H. Idei, T. Mutoh, and LHD experiment group, Recent upgrading of ECRH system and studies to improve ECRH performance in the LHD, EPJ Web Conf. 87, 02011 (2015).

[15] V. Erckmann, P. Brand, H. Braune, G. Dammertz, G. Gantenbein, W. Kasparek, H. P. Laqua, H. Maassberg, N. B. Marushchenko, G. Michel, M. Thumm, Y. Turkin, M. Weissgerber, A. Weller, W7-X ECRH Team at IPP Greifswald, W7-X ECRH Team at FZK Karlsruhe, and W7-X ECRH Team at IPF Stuttgart, Electron cyclotron heating for W7-X: Physics and technology, Fusion Sci. Technol. 52, 291 (2017).

[16] Y. Yoshimura et al., Stable sustainment of plasmas with electron internal transport barrier by ECH in the LHD, 60 , 025012 (2018).

[17] G. Fuchert et al., Increasing the density in Wendelstein 7-X: Benefits and limitations, Nucl. Fusion 60, 036020 (2020).

[18] K. Tanaka et al., Particle transport of LHD, Fusion Sci. Technol. 58, 70 (2010).

[19] Y. Ohtani, K. Tanaka, T. Tokuzawa, T. Akiyama, I. Yamada, R. Yasuhara, H. Funaba, M. Shoji, and M. Goto, Particle transport of electron cyclotron resonant heating plasma in Large Helical Device, 62, 025029 (2020).

[20] I. Yamada, K. Narihara, H. Funaba, T. Minami, H. Hayashi, T. Kohmoto, and LHD Experiment Group, Recent progress of the LHD thomson scattering system, Fusion Sci. Technol. 58, 345 (2010). 
[21] E. Pasch, M. N. A. Beurskens, S. A. Bozhenkov, G. Fuchert, J. Knauer, R. C. Wolf, and W7-X Team, The Thomson scattering system at Wendelstein 7-X, Rev. Sci. Instrum. 87, $11 E 729$ (2016).

[22] K. Tanaka, K. Kawahata, T. Tokuzawa, S. Okajima, Y. Ito, K. Muraoka, R. Sakamoto, K. Watanabe, T. Morisaki, H. Yamada, the LHD Experimental group, Density reconstruction using a multi-channel far-infrared laser interferometer and particle transport study of a pelletinjected plasma on the LHD, Plasma Fusion Res. 3, 050 (2008).

[23] M. Yoshinuma, K. Ida, M. Yokoyama, M. Osakabe, and K. Nagaoka, Charge-exchange spectroscopy with pitch-controlled double-slit fiber bundle on LHD, Fusion Sci. Technol. 58, 375 (2010).

[24] O. P. Ford et al., Charge exchange recombination spectroscopy at Wendelstein 7-X, Rev. Sci. Instrum. 91, 023507 (2020).

[25] W. I. van Rij and S. P. Hirshman, Variational bounds for transport coefficients in three-dimensional toroidal plasmas, Phys. Fluids B 1, 563 (1989).

[26] Y. Turkin, H. Maaßberg, C. D. Beidler, J. Geiger, and N. B. Marushchenko, Current control by ECCD for W7-X, Fusion Sci. Technol. 50, 387 (2006).

[27] W. Horton, D. Choi, and W. M. Tang, Toroidal drift modes driven by ion pressure gradients, Phys. Fluids 24, 1077 (1981).

[28] A. J. Brizard and T. S. Hahm, Foundations of nonlinear gyrokinetic theory, Rev. Mod. Phys. 79, 421 (2007).

[29] F. Jenko, W. Dorland, M. Kotschenreuther, and B. N. Rogers, Electron temperature gradient driven turbulence, Phys. Plasmas 7, 1904 (2000).

[30] T.-H. Watanabe and H. Sugama, Velocity-space structures of distribution function in toroidal ion temperature gradient turbulence, Nucl. Fusion 46, 24 (2005).

[31] M. Nunami, T.-H. Watanabe, and H. Sugama, Gyrokinetic Vlasov code including full three-dimensional geometry of experiments, Plasma Fusion Res. 5, 016 (2010).

[32] A. Ishizawa, T.-H. Watanabe, H. Sugama, M. Nunami, K. Tanaka, S. Maeyama, and N. Nakajima, Turbulent transport of heat and particles in a high ion temperature discharge of the Large Helical Device, Nucl. Fusion 55, 043024 (2015).

[33] M. A. Beer, S. C. Cowley, and G. W. Hammett, Field aligned coordinates for nonlinear simulations of tokamak turbulence, Phys. Plasmas 2, 2687 (1995).

[34] G. G. Plunk, P. Helander, P. Xanthopoulos, and J. W. Connor, Collisionless microinstabilities in stellarators. III. The ion-temperature-gradient mode, Phys. Plasmas 21, 032112 (2014).
[35] T.-H. Watanabe, H. Sugama, and M. Nunami, Effects of equilibrium-scale radial electric fields on zonal flows and turbulence in helical configurations, Nucl. Fusion 51, 123003 (2011).

[36] P. H. Diamond, S.-I. Itoh, K. Itoh, and T. S. Hahm, Zonal flows in plasma-a review, Plasma Phys. Controlled Fusion 47, R35 (2005).

[37] G. G. Plunk, P. Xanthopoulos, and P. Helander, Distinct Turbulence Saturation Regimes in Stellarators, Phys. Rev. Lett. 118, 105002 (2017).

[38] M. Nunami, T.-H. Watanabe, H. Sugama, and K. Tanaka, Linear gyrokinetic analyses of ITG modes and zonal flows in LHD with high ion temperature, Plasma Fusion Res. 6, 1403001 (2011).

[39] M. Nunami, T.-H. Watanabe, H. Sugama, and K. Tanaka, Gyrokinetic turbulent transport simulation of a high ion temperature plasma in large helical device experiment, Phys. Plasmas 19, 042504 (2012).

[40] M. Nakata, M. Nunami, H. Sugama, and T.-H. Watanabe, Isotope Effects on Trapped-Electron-Mode Driven Turbulence and Zonal Flows in Helical and Tokamak Plasmas, Phys. Rev. Lett. 118, 165002 (2017).

[41] F. Jenko and A. Kendl, Radial and zonal modes in hyperfinescale stellarator turbulence, Phys. Plasmas 9, 4103 (2002).

[42] P. Xanthopoulos, H. E. Mynick, P. Helander, Y. Turkin, G. G. Plunk, F. Jenko, T. Görler, D. Told, T. Bird, and J. H. E. Proll, Controlling Turbulence in Present and Future Stellarators, Phys. Rev. Lett. 113, 155001 (2014).

[43] H. E. Mynick, N. Pomphrey, and P. Xanthopoulos, Optimizing Stellarators for Turbulent Transport, Phys. Rev. Lett. 105, 095004 (2010).

[44] H. Mynick, P. Xanthopoulos, B. Faber, M. Lucia, M. Rorvig, and J. N. Talmadge, Turbulent optimization of toroidal configurations, Plasma Phys. Controlled Fusion 56, 094001 (2014).

[45] S. A. Bozhenkov et al., High-performance plasmas after pellet injections in Wendelstein 7-X, Nucl. Fusion 60, 066011 (2020).

[46] P. Xanthopoulos, S. A. Bozhenkov, M. N. A. Beurskens, H. M. Smith, G. G. Plunk, P. Helander, C. D. Beidler, J. A. Alcusón, A. Alonso, A. Dinklage, O. Ford, G. Fuchert, J. Geiger, J. H. E. Proll, M. J. Pueschel, Y. Turkin, F. Warmer, and the W7-X Team, Turbulence Mechanisms of Enhanced Performance Stellarator Plasmas, Phys. Rev. Lett. 125, 075001 (2020).

[47] H. Yamada, J. H. Harris, A. Dinklage, E. Ascasibar, F. Sano, S. Okamura, J. Talmadge, U. Stroth, A. Kus, S. Murakami, M. Yokoyama, C. D. Beidler, V. Tribaldos, K. Y. Watanabe, and Y. Suzuki, Characterization of energy confinement in net-current free plasmas using the extended international stellarator database, Nucl. Fusion 45, 1684 (2005). 